\title{
ADAPTIVE GRID REFINEMENT USING THE GENERALISED FINITE-DIFFERENCE METHOD
}

\author{
PAUL KEW
}

(Received 29 May 2020; first published online 11 September 2020)

2020 Mathematics subject classification: primary 65N50; secondary 76D05.

Keywords and phrases: Navier-Stokes equations, generalised finite-difference method, adaptive grid refinement method.

The combination of the generalised finite-difference method (GFDM) and adaptive grid refinement is applied to solve two-dimensional fluid flow problems. The accuracy of this combination is demonstrated by solving the two-dimensional lid-driven cavity flow and two-dimensional backward-facing step flow problems, and comparing the results against the benchmarks. This new computational fluid dynamics (CFD) formulation is applied to solve a two-dimensional meter flow application to determine the velocity profiles through the centre of the meter for higher Reynolds numbers.

To verify the accuracy of this combination, analytical two-dimensional and three-dimensional Laplace partial differential equations are solved by two methods. The first method uses the finite-difference method (FDM) over a uniform grid of nodes, and the second method uses the GFDM over a nonuniform grid of nodes. Computational cost and accuracy comparisons are made for both methods.

PAUL KEW, School of Computing and Mathematics, Charles Sturt University, Wagga Wagga, New South Wales 2678, Australia e-mail: pk210270@ hotmail.com

Thesis submitted to Charles Sturt University in August 2019; degree approved on 18 December 2019; principal supervisor Zhenquan Li, co-supervisors Philip Charlton and Michael Kemp.

(C) 2020 Australian Mathematical Publishing Association Inc. 